\title{
Acute Kidney Injury Frequency in Novel Coronavirus Pandemic and Infection Frequency in Maintenance Hemodialysis Patients
}

\section{Yeni Koronavirüs Pandemisinde Akut Böbrek Hasarı Sıklığı ve Hemodiyaliz Hastalarında Enfeksiyon Sıklı̆̆ı}

\author{
Kadir Gökhan Atılgan ${ }^{1}$, Ebru Gok Oguz ${ }^{1}$, Fevzi Coșkun Sökmen ${ }^{2}$, Fatma Ayerden Ebinç ${ }^{1}$, Gülay Ulusal \\ Okyay $^{1}$, Mehmet Deniz Aylı ${ }^{1}$, İran Sencan ${ }^{3}$ \\ ${ }^{1}$ Sağlık Bilimleri Üniversitesi Dışkapı Yıldırım Beyazıt Eğitim ve Araştırma Hastanesi, Nefroloji Kliniği \\ Ankara/Türkiye \\ ${ }^{2}$ Sağlık Bilimleri Üniversitesi Dr Abdurrahman Yurtaslan Ankara Onkoloji Eğitim ve Araştırma Hastanesi \\ Dahiliye Kliniği Ankara/Türkiye \\ ${ }^{3}$ Sağılı Bilimleri Üniversitesi Dışkapı Yıldırım Beyazıt Eğitim Ve Araştırma Hastanesi, Enfeksiyon Hastalıkları \\ Ve Klinik Mikrobiyoloji Kliniği Ankara/Türkiye
}

Dergiye Ulaşma Tarihi: Dergiye Kabul Tarihi: Doi: 10.5505/aot.2020.80947

\section{ÖZET}

GÍRIŞ ve AMAÇ: Türkiye, COVID-19'dan en fazla etkilenen iki kıta olan Avrupa ve Asya arasındaki köprüdür. $\mathrm{Bu}$ çalışma nefroloji bölümüne danışılan COVID-19 hastalarının prognozunu ve sonuçlarını paylaşmaktadır.

YÖNTEM ve GEREÇLER: 11 Mart 2020 ve 22 Nisan 2020 tarihleri arasında COVID-19 tanısı ile yatırılan hastaların kayıtları retrospektif olarak değerlendirildi ve bunlardan hemodiyaliz (HD) alan ve akut böbrek hasarı (ABH) tanısı alan hastalar bu çalışmaya dahil edildi.

BULGULAR: COVID-19 ile tedavi edilen 352 hasta çalışmaya dahil edildi. Bunlardan nefroloji bölümüne başvuran 16 hastanın 8'ine daha önce HD tedavisi uygulanırken COVID-19 teşhisi konuldu. Diğer 8 hasta COVID19 ile takip edilirken $\mathrm{ABH}$ tanısı aldı. Bunlardan $5 \mathrm{ABH}$ hastası yoğun bakım ünitesine alındı ve hepsi takip döneminde entübe edildi. Ancak ABH tanısı alan beş hasta öldü. Yoğun bakıma ihtiyaç duymayan üç ABH tanılı hasta taburcu edildi.

TARTIŞMA ve SONUÇ: Bu çalışma, ABH gelişen hastalarda COVID-19'un daha önce HD alan hastalara göre daha ölümlü olduğunu göstermektedir. Bunun COVID-19 ile ilişkili akut böbrek tutulumuna bağlı olabileceğini düşünüyoruz.

Anahtar Kelimeler: Akut böbrek hasarı, COVID-19, hemodiyaliz, yeni koronavirüs

\section{ABSTRACT}

INTRODUCTION: Turkey is the bridge between Europe and Asia, which are the two continents that are affected by COVID-19 the most. This study shares the prognosis and results of COVID-19 patients, who have been consulted to the department of nephrology.

METHODS: The records of patients who were hospitalized with the diagnosis of COVID-19 between 11 March 2020 and 22 April 2020 were evaluated retrospectively and of these, the patients with receiving hemodialysis (HD) and diagnosed with acute kidney injury (AKI) were involved in this study.

RESULTS: 352 patients who were treated with COVID-19 were included in this study. Of these, 8 of the 16 patients consulted to the nephrology department were diagnosed with COVID-19 while receiving HD treatment previously. The remaining 8 patients who were diagnosed with AKI while being followed-up for COVID-19. Of these, 5 patients with AKI were taken to intensive care unit and all of them were intubated in the follow-up period. But five patients diagnosed with AKI were died. Three patients with AKI who did not need intensive care were discharged.

DISCUSSION AND CONCLUSION: This study shows that COVID-19 was more mortal in patients who developed AKI compared to patients who previously received HD. We think that this may be due to acute renal involvement related to COVID-19.

Keywords: Acute kidney injury, COVID-19, hemodialysis, novel coronavirus 


\section{INTRODUCTION}

World Health Organization (WHO) announced the novel coronavirus infection disease(COVID-19) pandemic in March 2020. Although SARS-CoV-2 (Severe acute respiratory syndrome-Coronavirus-2), theofficial name of the novel coronavirus defined by the WHO, is a part of the SARS-CoV and MERS-CoV (Middle east respiratory syndrome-Coronavirus) families that we are familiar with, the novel coronavirus displays unique clinical results, thanks to the mutations. The coronavirus displays itself with respiratory system infections and may lead to multi-organ involvement and death[1].Concerns about the COVID-19 increase due to the absence of any known treatment or vaccine. Besides, comorbid diseases in patients with COVID-19 increase health problems.

Novel coronavirus infection requires a special treatment and action plan for nephrology patients, including dialysis, renal transplant, andchronic kidney disease (CKD) patients[2].However, there is only a limited number of clinical studies on the frequency and the clinical course of COVID-19, its relation with acute kidney injury (AKI) and COVID-19 frequency in maintenance hemodialysis (HD) patients, who constitute an important share of nephrology patients[3-5].Turkeyis among the high-risk countries since the country lies between Europe and Asia, which are the two continents that are affected by COVID-19 the most. This study analyzes the frequency and results of kidney involvement for patients that applied to our hospital in Ankara, which is considered as pandemic hospital, with COVID19.The study aims to identifythe relationship between COVID-19 and AKI and the frequency and the course of COVID-19 inHD patients.

\section{MATERIAL METHOD}

The study was carried out between 11 March 2020 and 22 April 2020. During the period, 849 patients were examined for COVID-19.352 patients, who were diagnosed with COVID-19 and hospitalized, were included to our study. 2019-nCoV Real Time PCR method was used for identification of SARS-CoV-2 in nasopharyngeal swabs. Chest X-ray and thoracic computed tomographywere used for evaluation of lower respiratory tract. Patients with positive clinical findings andPCR tests were considered as COVID-19and received medical treatment in line with the COVID-19 Guide, which was prepared by the Turkish Ministry of Health.

The study started by identifying the patients' intensive care need, prognosis and survive. We used themonitoring records of the patients that were diagnosed with COVID-19 to find out SARS-CoV-2 positivity via PCR method, intensive care need and the number of patients intubated and died. We recorded the baseline symptoms and findings, medical history and treatment, number of HD received, baseline therapy protocols, prognosis, routine serum biochemistry, hemogram, venous blood gas, NT-proBNP (Brain natriuretic peptide), creatine kinase, D-dimer, troponin-I (cTnI), procalcitonin, $\mathrm{C}$ reactive protein (CRP), ferritin and scanning test results for the patients, who were diagnosed either with COVID-19 during the period they received HD therapy due to endstage renal disease (ESRD)or with AKI while receiving COVID-19 therapy. AKI was diagnosed using KDIGO-2012 guidelines[6].The study was performed in accordance with Declaration of Helsinki. The study design was approved by local ethical committee (Approved date: 08.May.2020 and number: 87/04).

\section{RESULTS}


From the day that pandemic started until now, 849 patients were examined in our hospital for COVID-19. 352 patients that were hospitalized with COVID-19 diagnosis were recorded to the study. 2019-nCoV Real Time PCR Kit identified SARS-CoV-2 positivity in these 352 patients. 41 patients, who received COVID-19 therapy,were followed in intensivecare unit (ICU), whereas 25 (7.1\%) were intubated.27 patients $(7.67 \%)$ died during the process. 16 patients were diagnosed with either COVID-19 during the period they received HD therapy due to ESRD and or with AKI while receiving COVID-19 therapy.Median age of these 16 patients, who were referred to department of nephrology, was 61.5(35-81) yearsand 8 of them $(50 \%)$ were male. Mean hospitalization period was $7.63 \pm 3.70$ days. The most common baseline symptomswerecough, dyspnea and fever, respectively (Table 1).

Table 1:General futures of patients referred to nephrology

\begin{tabular}{|l|c|}
\hline Number of patients (n) & 16 \\
\hline $\begin{array}{l}\text { Number of patients receiving } \\
\text { maintenance HD (n) }\end{array}$ & 8 \\
\hline Number of patients with AKI (n) & 8 \\
\hline Age (years) & $\begin{array}{c}64.5(35- \\
81)^{*}\end{array}$ \\
\hline Men (n) & 8 \\
\hline Number of patients who smoke & 6 \\
\hline Recet travel orsick contact (n) & 1 \\
\hline SYMPTOMS & 11 \\
\hline Cough (n) & 7 \\
\hline Fever (n) & 11 \\
\hline Dyspnea (n) & 2 \\
\hline Nausea and vomiting (n) & 1 \\
\hline Diarrhea (n) & 3 \\
\hline Hypotansiyon (n) & \\
\hline
\end{tabular}

* Median (interquartile range)

HD: hemodialysis, AKI: Acute kidney injury

Eight patients (2.27\%) were diagnosed with ESRD and received HD therapy. Medianage of these patients was 58.5 (3571)years, two (25\%)were male and two patients had smoking history. Table 2 shows the characteristics of these patients. The analysis of comorbid diseasesshows that eight $(100 \%)$ had hypertension (HT) andthree (37.5\%) haddiabetes mellitus (DM). Patient \#1 was an Iranian citizen, who had contact with a relative that recently came from Iran. Other patients did not have recent travel or sick contact. Patient \#3was a scheduledHDpatient with renal transplant history, who was receivinglower dose immunosuppressive therapy after chronicallograft rejection. These patients, who received HDand did not have intensive care need, were monitored in COVID-19 isolation clinic. Mean hospitalization period was $8.50 \pm$ 3.67 days. All HD patients that were diagnosed with COVID-19 were discharged after anti-viral therapy. 
Table 2 : Demographics and baseline laboratory data of hemodialysis patients with COVID-19

\begin{tabular}{|c|c|c|c|c|c|c|c|c|c|}
\hline Case/Age Sex & Comorbidity & $\begin{array}{l}\text { Symptom } \\
\text { findings. }\end{array}$ & $\begin{array}{l}\text { RAS/ } \\
\mathrm{OAD}\end{array}$ & Thoracic CT & $\begin{array}{l}\text { WBC/Ned/ } \\
\mathrm{Lvm} / \mathrm{NLR}\end{array}$ & $\begin{array}{l}\text { CK LDH } / \\
\text { Ferr/CRP }\end{array}$ & $\begin{array}{l}\mathrm{DD} / \mathrm{PRC} / \mathrm{sTnI} / \\
\mathrm{BNP} / \text { Fjen. }\end{array}$ & $\begin{array}{l}\text { Treatment/ } \\
\text { hospitalization period }\end{array}$ & Qutcome \\
\hline $\begin{array}{l}\text { Patient } \# 1 \\
55 \text { yo/F }\end{array}$ & $\begin{array}{l}\text { HT, CVO, } \\
\text { ESRD }\end{array}$ & $\begin{array}{l}\text { Cough, fexer, } \\
\text { dyspnea, }\end{array}$ & $-/$ & $\begin{array}{l}\text { Ground glass } \\
\text { opacity. } \\
\text { Pneumonia }\end{array}$ & $\begin{array}{l}8.63 / 6.46 / \\
1.09 / 5.93\end{array}$ & $\begin{array}{l}79 / 167 / \\
2676 / 7.15\end{array}$ & & $\begin{array}{l}\text { HCQ-Q seltamixi- } \\
\text { Meropenem- } \\
\text { linezolid / 14days }\end{array}$ & Discharged \\
\hline $\begin{array}{l}\text { Patient } \# 2 \\
57 \text { yo } / F\end{array}$ & $\begin{array}{l}\text { HT, DM, } \\
\text { FMF, ESRD }\end{array}$ & Cough & 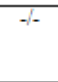 & $\begin{array}{l}\text { Ground glass } \\
\text { spacity } \\
\text { Pneumonia }\end{array}$ & $\begin{array}{l}4.09 / 3.36 / \\
0.46 / 7.30\end{array}$ & $\begin{array}{l}57 / 321 / \\
2001 / 22.09\end{array}$ & $-/ 79.44 / 2 /-$ & $\begin{array}{l}\text { HCQ-O seltamivir- } \\
\text { Azithromycin } / 7 \text { davs }\end{array}$ & Discharged \\
\hline $\begin{array}{l}\text { Patient } \# 3 \\
63 \text { yo } F\end{array}$ & $\begin{array}{l}\text { HT, RTX, } \\
\text { ESRD }\end{array}$ & $\begin{array}{l}\text { Cough, fever, } \\
\text { dyspnea, } \\
\text { neutropenia }\end{array}$ & $\%$ & $\begin{array}{l}\text { Ground glass } \\
\text { spacity } \\
\text { Pneumonia }\end{array}$ & $\begin{array}{l}0.35 / 0.21 / \\
0.13 / 1.62\end{array}$ & $\begin{array}{l}29 / 456 / \\
1310 / 137,6\end{array}$ & $\begin{array}{l}1.06 / 0.09 / 0.09 / \\
549.3 / 436\end{array}$ & $\begin{array}{l}\text { HCQ-Q seltamivir- } \\
\text { Meropenem- } \\
\text { limezolid / 14days }\end{array}$ & Discharged \\
\hline $\begin{array}{l}\text { Patient } \# 4 \\
60 \text { yo } / \mathrm{F}\end{array}$ & $\begin{array}{l}\text { HT,DM, } \\
\text { ESRD }\end{array}$ & $\begin{array}{l}\text { Cough, fever, } \\
\text { dyspnea, }\end{array}$ & $-\gamma$ & $\begin{array}{l}\text { Ground glass } \\
\text { opacity } \\
\text { Pneumonia }\end{array}$ & $\begin{array}{l}8.58 / 6.07 / / \\
1.1 / 5.5\end{array}$ & $\begin{array}{l}219 / 208 / \\
156 / 285\end{array}$ & $\begin{array}{l}-/ / 0.25 / \\
21316 /-\end{array}$ & $\begin{array}{l}\text { HCQ-O seltamivi- } \\
\text { Cephalosporin- } \\
\text { Clarithromvcin } 7 \text { days }\end{array}$ & Discharged \\
\hline $\begin{array}{l}\text { Patient } \# 5 \\
51 \text { yo } F\end{array}$ & HT, ESRD & $\begin{array}{l}\text { Cough, fexer, } \\
\text { nausea, } \\
\text { vomiting }\end{array}$ & $-1-$ & $\begin{array}{l}\text { Ground glass } \\
\text { spacity } \\
\text { Pneumonia }\end{array}$ & $\begin{array}{l}9.63 / 8.25 / \\
0.7 / 11.79\end{array}$ & $\begin{array}{l}40 / 239 / \\
751 / 51\end{array}$ & $\begin{array}{l}0.61 / 0.65 / 0.1 / \\
35000 / 544\end{array}$ & $\begin{array}{l}\text { HCQ-Q seltamivi- } \\
\text { Lexofloxacin } 7 \text { days }\end{array}$ & Discharged \\
\hline $\begin{array}{l}\text { Patient } \# 6 \\
71 \text { yo:M }\end{array}$ & $\begin{array}{l}\text { HT, CAD, } \\
\text { COPD, ESRD }\end{array}$ & $\begin{array}{l}\text { Fever, } \\
\text { hypotension }\end{array}$ & $-/$ & $\begin{array}{l}\text { Ground glass } \\
\text { opacity } \\
\text { Pneumonia }\end{array}$ & $\begin{array}{l}15.3 / 12 / \\
1.78 / 6.76\end{array}$ & $\begin{array}{l}86 / 188 / \\
1100 / 167\end{array}$ & $\begin{array}{l}0.59 / 3.76 / 1.51 / \\
29066 / 686\end{array}$ & $\begin{array}{l}\text { HCQ-Oseltamivir } \\
\text { 3daxs.Meropenem- } \\
\text { linezolid / 14days }\end{array}$ & Discharged \\
\hline $\begin{array}{l}\text { Patient } \# 7 \\
35 \mathrm{yo} / \mathrm{M}\end{array}$ & HT, ESRD & $\begin{array}{l}\text { Cough, } \\
\text { dyspnea, }\end{array}$ & $+/-$ & $\begin{array}{l}\text { Ground glass } \\
\text { opacity } \\
\text { Pneumonia } \\
\text { Pleural effusion }\end{array}$ & $\begin{array}{l}9.63 / 7.66 \\
1.12 / 6.84\end{array}$ & $\begin{array}{l}249 / 317 / \\
357 / 205\end{array}$ & $\begin{array}{l}0.74 / 1.32 / 0.1 / \\
35000 / 565\end{array}$ & $\begin{array}{l}\text { HCQ-Favipravir- } \\
\text { Azithromucin- } \\
\text { Meropenem- } \\
\text { linezolid / } 14 \text { days }\end{array}$ & Discharged \\
\hline $\begin{array}{l}\text { Patient } \# 8 \\
69 \text { yo } F\end{array}$ & $\begin{array}{l}\text { HT, DM, } \\
\text { CAD, CHF }\end{array}$ & Cough, & $+/+$ & $\begin{array}{l}\text { Ground glass } \\
\text { opacity. } \\
\text { Pneumonia } \\
\text { Pleural effusion }\end{array}$ & $\begin{array}{l}17.5 / 15.2 / \\
1.00 / 15.2\end{array}$ & $\begin{array}{l}32 / 166 / \\
1563 / 344\end{array}$ & $\begin{array}{l}1.06 / 4.81 / 1.43 / \\
32404 / 713\end{array}$ & $\begin{array}{l}\text { HCQ-Oseltamivit- } \\
\text { Cephalosporim- } \\
\text { Clarithromycin } 7 \text { days }\end{array}$ & Discharged \\
\hline
\end{tabular}

HT: Hypertension, CVO: Cerebrovascular disease, DM: Diabetes mellitus, FMF: Familial mediterrean fever, RTx: Renal transplantation CAD: Coronary artery disease, COPD:Chronic obstructive pulmonary disease,CHF: Congestive heart failure,ESRD: End stage renal disease, RAS: Renim-angiotensin system blocers, OAD:Oral anti-diabetic drugs, Cr: creatinine, Na: Sodium K: Potassium. WBC: White blood cell, Neu: neutrophile, Lvm: Lymphocyte, NLR: Neutrophile to lymphocyte ratio, CK: Creatin kinase, LDH: Lactate dehydrogenase, Ferr: Ferritim, CRP: C reactive protein, DD: DD imer, PRC: procalcitonin, sTnI: Troponin-I, NTproBNP: NT pro-brain natruretic peptide, Fien: Fibrinegen, CT: Computed tomography, HCQ: Hudroxychloroquine

The remaining 8patients(2.27\%) were diagnosed with AKI during the period they receivedCOVID-19 therapy and were referred to the department of nephrology. Median age of these patients was $66(37-81)$ years and $6(75 \%)$ were male. Four of the six male patients had smoking history.Mean hospitalization period was $6.57 \pm 4.04$ days. Table 3 shows the number of HD season and the results of baselinekidney function test. Level of proteinuria was +1 for four patients and +3 for one patient, who had HT, DM and CKDcomorbidity. Hematuria was evident in three patients and was at the levels of +3 and +4 . The number of patients diagnosed with AKI at the ICU was five (12.2\%) and the number of intubated patients diagnosed with AKI was five $(20 \%)$. The number of patients diagnosed with AKI among the dead patients wasfive (18.5\%).Table 3 also shows information on the patients' prognosis and survive. The analysis of the comorbid diseases shows that 6 patients $(75 \%)$ had HT, $6(75 \%)$ had DM, $4(50 \%)$ had coronary artery disease, 3 (37.5\%) had congestive heart failure, $3(37.5 \%)$ had CKD, $1(12.5 \%)$ had cerebrovascular accident, and $1(12.5 \%)$ had liver cirrhosis. Fiveof the intubated patientswith AKI died during their follow at the ICU. Median age of these patients was 64 (37-81) and mean hospitalization period was $6.40 \pm 4.93$ days.Threepatients were discharged. Hypertension, DM and CKD were the comorbid diseases of the discharged patients. Prisoner/patient (Patient \#12) was discharged with the decision to receive three HD sessions per week.Temporary HD catheters of the two other patientswere removed and they were discharged to be followed up at the outpatientsclinic with their basal creatinine levels after 14 days of isolation. Cytokine levels dramatically increased for only the patient \#16, who had no comorbid disease. Continuous renal replacement therapy (CRRT) was given to the patient, who receivedExtracorporeal membrane oxygenation (ECMO)support. Interleukin-6 inhibitor (Tocilizumab) was added to therapy. The patient died on the second hospitalization day (Table 3).

\section{DISCUSSION}

Hemodialysis patients are under high risk in terms of COVID-19 and its complications. These patients are inclined to infection due to the consequences of ESRD, including neutrophil, monocyte dysfunction, and deteriorationin T-cell activationsand humoral response[7]. They are mostly older and have two or more comorbid diseases. Repeating 
Table 3: Demographics and baseline laboratory data of COVID-19 patients referred to acute kidney injury

\begin{tabular}{|c|c|c|c|c|c|c|c|c|c|c|c|}
\hline $\begin{array}{l}\text { Case'Age/ } \\
\text { Sex }\end{array}$ & Comorbidity & $\begin{array}{l}\text { Symptom/ } \\
\text { findings, }\end{array}$ & $\begin{array}{l}\text { RAS/ } \\
\text { OAD }\end{array}$ & $\begin{array}{l}\text { Number } \\
\text { of HD } \\
\text { session }\end{array}$ & $\begin{array}{l}\text { Urea } \mathrm{Cr} / \\
\mathrm{Na} / \mathrm{K}\end{array}$ & Theracic CT & $\begin{array}{l}\text { WBC/Neu/ } \\
\mathrm{Lxm} / \mathrm{NLR}\end{array}$ & $\begin{array}{l}\text { CK/ LDH/ } \\
\text { Ferr/CRP }\end{array}$ & $\begin{array}{l}\mathrm{DD} / \mathrm{PRC} / \mathrm{cT} \text { ThI } \\
\text { BNP/Fjen。 }\end{array}$ & $\begin{array}{l}\text { Treatment' } \\
\text { hospitalization period }\end{array}$ & Outcome \\
\hline $\begin{array}{l}\text { Patient } \# 9 \\
66 \text { yo/M }\end{array}$ & $\begin{array}{l}\text { HT, DM, } \\
\text { CAD, CHF, } \\
\text { CKD }\end{array}$ & $\begin{array}{l}\text { Cough, } \\
\text { dyspnea, }\end{array}$ & $\gamma$ & 3 & $\begin{array}{l}121.6 / \\
3.52 / 133 / \\
5.12\end{array}$ & $\begin{array}{l}\text { Ground glass } \\
\text { spacity } \\
\text { Pneumonia } \\
\text { Pleural effusion }\end{array}$ & $\begin{array}{l}5.85 / 4.69 / \\
0.83 / 5.65\end{array}$ & $\begin{array}{l}109 / 316 / \\
498 / 196\end{array}$ & $\begin{array}{l}2.18 / 0.56 / 0.17 / \\
35000 /-\end{array}$ & $\begin{array}{l}\text { HCQ-Q seltamixir- } \\
\text { Levofloxacin / 7days }\end{array}$ & Discharged \\
\hline $\begin{array}{l}\text { Patient }=10 \\
66 \text { yo/M }\end{array}$ & $\begin{array}{l}\text { HT, DM, } \\
\text { CKD }\end{array}$ & $\begin{array}{l}\text { Cough, } \\
\text { fexer, } \\
\text { dyspnea, }\end{array}$ & $\%$ & 6 & \begin{tabular}{l|}
$163.8 /$ \\
$4.45 / 138 /$ \\
6.05
\end{tabular} & $\begin{array}{l}\text { Ground glass } \\
\text { opacity } \\
\text { Pleural effusion }\end{array}$ & $\begin{array}{l}18.6 / 16.6 / \\
0.76 / 21.87\end{array}$ & $\begin{array}{l}99 / 381 / \\
1067 / 446,7\end{array}$ & $\begin{array}{l}-/ / / 2.37 / / \\
35000 /-\end{array}$ & $\begin{array}{l}\text { HCQ-O seltamivit } \\
\text { Cephalosporin- } \\
\text { Clarithromycin/ 7days }\end{array}$ & Discharged \\
\hline $\begin{array}{l}\text { Patient } \# 11 \\
81 \text { yo/F }\end{array}$ & $\begin{array}{l}\text { HT, DM, } \\
\text { CAD } \\
\text { Alzheimer } \\
\text { Disease }\end{array}$ & $\begin{array}{l}\text { Dyspnea, } \\
\text { hypotension }\end{array}$ & $\%$ & 1 & $\begin{array}{l}262 / 4.97 / \\
152 / 5.33\end{array}$ & $\begin{array}{l}\text { Ground glass } \\
\text { opacity } \\
\text { Pneumonia } \\
\text { Pleural effusion }\end{array}$ & $\begin{array}{l}0.35 / 0.21 / \\
0.13 / 1.62\end{array}$ & $\begin{array}{l}2272 / 456 / \\
1310 / 137,6\end{array}$ & $\begin{array}{l}2.22 / 4.89 / 1.25 \\
35000 / 713\end{array}$ & $\begin{array}{l}\text { Faviptavi- } \\
\text { Meropenem- } \\
\text { linezolid / 2days }\end{array}$ & $\begin{array}{l}\text { Died } \\
\text { Intubated }\end{array}$ \\
\hline $\begin{array}{l}\text { Patient }=12 \\
51 \text { yo/M }\end{array}$ & $\begin{array}{l}\text { HT, DM, } \\
\text { CKD }\end{array}$ & Dyspnea & $-F$ & 4 & $\begin{array}{l}176 / 5.08 / \\
133 / 5.81\end{array}$ & $\begin{array}{l}\text { Ground glass } \\
\text { opacity } \\
\text { Pneumonia } \\
\text { Pleural effuusion }\end{array}$ & $\begin{array}{l}7.12 / 5.5 / \\
0.52 / 10.58\end{array}$ & $\begin{array}{l}119 / 154 / \\
69.6 / 9.15\end{array}$ & $\begin{array}{l}6.88 / 0.28 / 0.1 / \\
15545 / 362\end{array}$ & $\begin{array}{l}\text { HCQ-O seltamivir- } \\
\text { Cephalosporim- } \\
\text { Clarithromycin/ 7days }\end{array}$ & $\begin{array}{l}\text { Discharged } \\
\text { HD } 3 / 7\end{array}$ \\
\hline $\begin{array}{l}\text { Patient }=13 \\
60 \text { yo } M\end{array}$ & $\begin{array}{l}\text { HT, DM, } \\
\text { CAD, CHF, }\end{array}$ & $\begin{array}{l}\text { Cough, } \\
\text { dyspnea }\end{array}$ & $+/ 2$ & 1 & $\begin{array}{l}26 / 0.90 / \\
138 / 5.74\end{array}$ & $\begin{array}{l}\text { Ground glass } \\
\text { opacity } \\
\text { Pneumonia } \\
\text { Pleural effusion }\end{array}$ & $\begin{array}{l}18.0 / 8.8 / \\
6.97 / 1.26\end{array}$ & $\begin{array}{l}101 / 242 / \\
123 / 3.64\end{array}$ & $\begin{array}{l}2.08 / 0.08 / 4.95 / \\
662 / 542\end{array}$ & $\begin{array}{l}\text { HCQ-Favipravir- } \\
\text { Levofloxacin - } \\
\text { Meropenem- } \\
\text { linezolid / 14days }\end{array}$ & $\begin{array}{l}\text { Died } \\
\text { Intubated }\end{array}$ \\
\hline $\begin{array}{l}\text { Patient }=14 \\
75 \text { yo/ } \mathrm{F}\end{array}$ & $\begin{array}{l}\text { HT, CVO, } \\
\text { CAD, CHF }\end{array}$ & $\begin{array}{l}\text { Dyspnea, } \\
\text { hypotension } \\
\text {, diarrhea }\end{array}$ & -1 & 2 & $\begin{array}{l}56 / 0.8 / \\
131 / 5.75\end{array}$ & $\begin{array}{l}\text { Ground glass } \\
\text { opacity } \\
\text { Pneumonia } \\
\text { Pleural effusion }\end{array}$ & $\begin{array}{l}7.4 / 6.59 / \\
0.33 / 19.97\end{array}$ & $\begin{array}{l}83 / 566 / \\
395 / 15.71\end{array}$ & $\begin{array}{l}1.16 / 0.09 / 0.1 / \\
5049.6 / 533\end{array}$ & $\begin{array}{l}\text { Favipravir- } \\
\text { Lewofloxacin - } \\
\text { Meropenem- } \\
\text { linezolid / 7days }\end{array}$ & $\begin{array}{l}\text { Died } \\
\text { Intubated }\end{array}$ \\
\hline $\begin{array}{l}\text { Patient }=15 \\
64 \text { yo/M }\end{array}$ & $\begin{array}{l}\text { DM, Liver } \\
\text { cirrhosis. }\end{array}$ & Dyspnea, & $-1+$ & 4 & $\begin{array}{l}188 / 2.37 \\
126 / 3.03\end{array}$ & $\begin{array}{l}\text { Ground glass } \\
\text { spacity } \\
\text { Pneumpria }\end{array}$ & $\begin{array}{l}7.45 / 5.54 / \\
0.98 / 5.65\end{array}$ & $\begin{array}{l}219 / 558 / \\
217 / 126,43\end{array}$ & $\begin{array}{l}7.99 / 0.15 / 0.1 / \\
224.5 / 397\end{array}$ & $\begin{array}{l}\text { HCQ-Favipravir- } \\
\text { Cephalosporin- } \\
\text { Clarithromycin/ 7days }\end{array}$ & $\begin{array}{l}\text { Died } \\
\text { Intubated }\end{array}$ \\
\hline $\begin{array}{l}\text { Patient } \# 16 \\
37 \text { yo/M }\end{array}$ & - & $\begin{array}{l}\text { Cough, } \\
\text { fever, } \\
\text { dyspnea }\end{array}$ & $-\gamma$ & 1 & \begin{tabular}{l|}
$43 / 1,1 /$ \\
$139 / 4.32$
\end{tabular} & $\begin{array}{l}\text { Ground glass } \\
\text { spacity } \\
\text { Pneumonia }\end{array}$ & $\begin{array}{l}29.1 / 25.0 / \\
1.47 / 16.99\end{array}$ & $\begin{array}{l}710 / 465 / \\
362 / 397,5\end{array}$ & $\begin{array}{l}1.61 / 4.26 / 0.44 / \\
7718 / 737\end{array}$ & $\begin{array}{l}\text { HCQ-Favipravir- } \\
\text { Meropenem- } \\
\text { linezolid - } \\
\text { Tosiluzumab/2days }\end{array}$ & $\begin{array}{l}\text { Died } \\
\text { Intubated }\end{array}$ \\
\hline
\end{tabular}

F: Female, M: Male, HT: Hypertension, CVO: Cerebroyascular disease, DM: Diabetes mellitus, , CAD: Coronary artery disease, COPD: Chronic obstructive pulmonary disease CHF: Congestive heart failure, CKD: Chronic kidney disease, RAS: Renin-angiotensin system blocers, OAD: Oral anti-diabetic drugs, Cr: creatinine, Na: Sodium, K: Potassium, WBC: White blood cell, Neu: neutrophile, Lym: Lymphocyte, NLR: Neutrophile to lymphocyte ratio, CK: Creatin kinase, LDH: Lactate dehydrogenase, Ferr: Ferritin. CRP: C reactive protein, DD: DDimer, PRC: procalcitonim, sTnI: Troponin-I, NTproBNP: NT pro-brain natruretic peptide, Fien: Fibrimogen, CT: Computed tomography, HCQ: Hydroxuchloroquine

visits of HD patients to the HD center and close distance between the patients during the session are the other factors that increase the risk for COVID-19.

The percentage of HD patients among the patients that received COVID-19 therapy in our hospital was $2.27 \%$.No study that has a large population of dialysis patients with COVID-19 has been published yet. Wang et al. presented their experiences with fiveHD patients, who developed COVID-19 pneumonia at a university hospital that had 201 HD patients[8]. Following the first COVID-19 incidence, the researchers used PCR for diagnosis of all patients and found that the prevalence of COVID-19in the dialysis center was $2.5 \%$. Fu et al.used Oseltamivir and Umifenovir for successful treatment of a 75years old end stage kidney disease patient infected with novel coronavirus[9]. In our study, all HD patients with COVID-19 diagnosis were discharged after anti-viral therapy without ICU need.

Some of the studies note diarrhea as an atypical clinical signin COVID-19[8,10]. Most common symptoms in our study were cough, dyspnea and fever, respectively (Table 1). Only one patient was hospitalized with the symptom of diarrhea. Hypotension, which was evident in four patients in our study, was another atypical symptom of COVID-19.First case, Patient \#6 applied to our emergency service, stating that adequate ultrafiltration could not be performed at the dialysis center in the last week due to fever and hypotension. Blood pressure was normotensive in emergency room. X-ray taken at the emergency care unit showed no pleural or pericardial effusion. Ultrafiltration was not carried out during HD. We started inotropic infusion with the second HD session due to hypotension and sinus tachycardia. Acute coronary syndrome was eliminated due to EKG and clinical findings. There was no finding other than left ventricular hypertrophy in echocardiography and cardiac valve structure was normal. Inotropic infusion need decreased starting with the 10th day of therapy. Inotropic infusion was stopped on the 12th day of therapy and the patient was discharged after a two-day follow-up (Table 2). Since the patient responded to inotropic infusion, we did not plan further therapy (intraaortic balloon pump, intravenous immunoglobulin, prednisolone, 
ECMO). Given the possibility of infection, we did not carry out angiography and endomyocardial biopsy intervention since they were not necessity. Myocarditis definition of the biopsy was undetermined.Second case, Patient \#14 had clinical deterioration, decrease in oxygen saturation and hypotensionon the fourth hospitalization date. creatine kinase, creatine kinase -mb, cTnI and NT-proBNP levels elevated to $2410 \mathrm{U} / \mathrm{L}, 215 \mathrm{U} / \mathrm{L}, 1.85$ $\mathrm{ng} / \mathrm{ml}$, and $15000 \mathrm{ng} / \mathrm{L}$, respectively. There was no symptoms of acute coronary syndrome other than the existing atrial fibrillation in EKG. The patient died on the third day of the start of inotrope infusion. Advanced intervention could not be made himdue to the existing clinical deterioration and comorbidities. Third and fourth cases, Patient\#11 and \#16, who admitted to our hospital with hypotension, died on the second hospitalization day. Table 3 shows the results of both all three patients.

The clinical presentation of patients with acute myocarditis varies, ranging from a subclinical disease to a fulminant heart failure and cardiogenic shock. A post-mortem analysis on 20 patients with SARS found viral RNA of SARS-CoVin the hearth tissue of seven (35\%) patients[11]. SARS-CoV may result withmyocardial inflammation and damage by leading to a decrease in angiotensin converting enzyme 2 (ACE2) expression. Besides, Alhogbani defined acute myocarditis related with MERS-CoV[12].Case presentations on cardiac involvement in COVID-19 was published[13].In addition to cardiac involvement direct from ACE2, cytokine release syndrome or myocardial cell apoptosis caused by hypoxia-induced excessive intracellular calcium may be the underlying mechanisms[14].We believe that unexplained hypotension in HD patients during pandemic may also be considered as COVID-19associated cardiac involvement (acute viral myocarditis or myocardial inflammation).
There are different results on the frequency of AKI in COVID-19 patients. There is a meaningful difference between the patients at the ICU with and without severe pulmonary involvement in terms of the frequency of AKI. The frequency of AKI for patients without serious diseases ranges between $0.5 \%$ and $15 \%$ whereas the range for the patients that had serious diseases is between $18 \%$ and $37.5 \%$ [15].Among the patients that received COVID-19 therapy in our study, the frequency of AKI was $2.27 \%$ (8 patients), the number of patients with AKI that were followed-up at the ICU was $5 / 41$ (12.2\%), the number of intubated patients with AKI was 5/25 (20\%) and the number of patients with AKI among the patients that died was $5 / 27(18.5 \%)$.

We tried to find out whether the kidneys of patients with COVID-19 are primarily affected by the disease or by multiple organ dysfunction.Novel coronavirusbinding tohuman ACE2 as a cell surface receptor. Although the disease primarily affects lower respiratory tract, it may be attached to other organs. High ACE2 expression was identified in type II alveolar cells, myocardialcells, kidney proximal tubule cells, ileum and esophagus epithelial cells and bladder urothelial cells. Due to this reason, these organs and tissues are the targets for SARS-CoV-2. In the kidneys, ACE2 is expressed in the apical brush borders of proximal tubules and the podocytes in less density. SARS-CoV-2 RNA was found in the urine of the patients with COVID-19[16]. Since we did not have the kit, we did not analyze SARS-CoV-2 in urine sedimentary. Postmortem analysis of Su et al. on 26 patients with COVID-19 in China found severe acute tubular injury, endothelial injury, occlusion of microvascular lumens, direct renal parenchymal infection, glomerular and vascular findings related with DM and HT. Electron microscopy observations identifiednovel coronavirus particles in the tubular epithelium and the podocytes[17]. The findings of these studies may lead us to suggest that COVID-19 may 
cause AKI by direct renal damage. Prospective study of Cheng et al. on 701 COVID-19 patients found elevated serum creatinine, proteinuria and hematuria in $14.4 \%, 43.9 \%$ and $26.7 \%$ of the patients. Age, elevated baseline serum creatinine and blood urea, proteinuria, hematuria and AKI were independent risk factors for in-hospital death[15]. In our study, there was proteinuriain five patients andhematuriain three patients. $5(20 \%)$ of the 25 patients that were followed-up at the ICU had received HD therapy due to AKI. 5 (18.5\%) of the $27(7.67 \%)$ patients that died had AKI.

The same study[15]found elevated leukocyte count, decreased lymphocyte and platelet count, severe coagulation disorders, and elevated D-dimer level in patients with elevated creatinine levels. Besides, aspartate aminotransferase, lactate dehydrogenase and procalcitoninlevels were higher for these patients. Analysis of the laboratory characteristics of the patients that need HD therapy due to AKI shows a striking increase in CRP (Patients \#9,10,11,15 and 16) and ferritin (Patients\#9, 10 and 11) levels compared to the acute phase reactants (Table 3). Significant leukocytosis was evident in patients \#10, 13 and 16 and lymphopenia was rare in these patients. With the exception of two patients, baseline neutrophil and lymphocyte was high. Baseline cTnI(Patients \#10, 11, 13 and 16), D-dimer (Patients \#9, 11, 12,13 and15), lactate dehydrogenase (Patients \#9,10,11,14,15 and16), procalcitonin (Patient \#11, 16) and NTproBNP (Patients \#9,10,11, 12,14 and 16) was high. Table 3 shows the values.

We had one patient (Patient \#16) that received CRRT.CRRT may be a better option for the treatment of COVID-19 infected AKI patients, who have poor hemodynamic status during dialysis or receive inotropic agent and mechanical ventilator support. Burgneret al. stated that CRRTin a patient on ECMO can be done by adding an in-line hemofilterinto the ECMO circuit[18]. CRRT can be usedforsepsis treatment due todecreasing inflammatory response by using various filters. Althoughthese membranes may be helpful in case of cytokine release syndrome that may occur during the course of the disease, the positive effects of these costly membranes for the COVID-19 patients has not been proven yet.

\section{CONCLUSION}

Given that all scheduled HD patients in our study were discharged without ICU need, we may suggest that they had a milder COVID-19 despite the fact that they are considered immunosuppressive. According to the results of our hospital, treatments can be provided in scheduled HD patients without complication in COVID-19.Hemodialysispatients with COVID-19 may have atypicalsymptoms other thandiarrhea, such as hypotension. During the pandemic, we should also consider the possibility of myocardial inflammation related with COVID-19in HD patients who develop hypotension. As a result, there is a relationship between AKI and morbidity and mortality in ICU patients diagnosed with COVID-19.

\section{Çıkar Çatışması: Yok}

\section{References}

1. Huang C, Wang Y, Li X, et al. Clinical features of patients infected with 2019 novel coronavirus in Wuhan, China. Lancet. 2020;395(10223):497-506.

2. Naicker S, Yang CW, Hwang SJ, Liu BC, Chen JH, Jha V. The Novel Coronavirus 2019 epidemic and kidneys. Kidney Int.2020;97(5):824-828.

3. Basile C, Combe C, Pizzarelli F, et al. Recommendations for the prevention, mitigation and containment of the emerging SARS-CoV-2 (COVID-19) pandemic in haemodialysis centres. Nephrol Dial Transplant. 2020;35(5):737-741

4. Chu KH, Tsang WK, Tang CS, et al. Acute renal impairment in coronavirus-associated severe acute respiratory syndrome. Kidney Int.2005;67(2):698-705.

5. $\mathrm{Li} \mathrm{J}, \mathrm{Xu}$ G. Lessons from the Experience in Wuhan to Reduce Risk of COVID-19 Infection in Patients 
Undergoing Long-Term Hemodialysis. Clin J Am SocNephrol.2020;15(5):717-719

6. Khwaja A. KDIGO clinical practice guidelines for acute kidney injury. Nephron Clin Pract. 2012;120(4):c179-184.

7. Ikizler TA. COVID-19 and Dialysis Units: What Do We Know Now and What Should We Do? Am J Kidney Dis. 2020;76(1):1-3.

8. Wang R, Liao C, He H, et al. COVID-19 in Hemodialysis Patients: A Report of 5 Cases. Am J Kidney Dis. 2020;76(1):141-143.

9. Fu D, Yang B, Xu J, Mao Z, Zhou C, Xue C. COVID-19 Infection in a Patient with End-Stage Kidney Disease. Nephron. 2020;144(5):245-247.

10. Ferrey AJ, Choi G, Hanna RM, et al. A Case of Novel Coronavirus Disease 19 in a Chronic Hemodialysis Patient Presenting with Gastroenteritis and Developing Severe Pulmonary Disease. Am J Nephrol. 2020;51(5):337-342.

11. Oudit GY, Kassiri Z, Jiang C, et al. SARS-coronavirus modulation of myocardial ACE2 expression and inflammation in patients with SARS.Eur J Clin Invest. 2009;39(7):618-25.
12. Alhogbani T. Acute myocarditis associated with novel Middle east respiratory syndrome coronavirus. Ann Saudi Med. 2016;36(1):78-80.

13. Fried JA, Ramasubbu K, Bhatt R, et al. The Variety of Cardiovascular Presentations of COVID-19. Circulation. 2020;141(23):1930-1936.

14. Zheng YY, Ma YT, Zhang JY, Xie X. COVID-19 and the cardiovascular system. Nat Rev Cardiol. 2020;17(5):259260.

15. Cheng Y, Luo R, Wang $\mathrm{K}$, et al. Kidney disease is associated with in-hospital death of patients with COVID19. Kidney Int. 2020;97(5):829-838.

16. Sun J, Zhu A, Li H, et al. Isolation of Infectious SARSCoV-2 from Urine of a COVID-19 Patient. Emerg Microbes Infect. 2020;9(1):991-993.

17. Su H, Yang M, Wan C, et al. Renal histopathological analysis of 26 postmortem findings of patients with COVID-19 in China. Kidney Int. 2020;98(1):219-227.

18. Burgner A, Ikizler TA, Dwyer JP. COVID-19 and the Inpatient Dialysis Unit: Managing Resources During Contingency Planning Pre-Crisis. Clin J AmSoc Nephrol. 2020;15(5):720-722. 